\section{Associações entre medidas ecocardiográficas e função renal em hipertensos na Atenção Básica}

\author{
Echocardiographic associations with renal function in hypertensive \\ individuals treated at Primary Care level
}

\section{Autores}

Fernando Alberto Costa Cardoso da Silva ${ }^{1}$

Vinícius José da Silva

Nina $^{2}$

Alcione Miranda dos

Santos $^{2}$

José Albuquerque Figueredo $\mathrm{Neto}^{2}$

Antonio Augusto Moura da Silva ${ }^{2}$

Francival Leite de Sousa ${ }^{1}$

Eduardo Durans Figueredo ${ }^{1}$

Natalino Salgado Filho

${ }^{1}$ Hospital Universitário da Universidade Federal do Maranhão - HUUFMA.

¿Universidade Federal do

Maranhão - UFMA.

Data de submissão: 10/12/2011 Data de aprovação: 06/01/2012

\section{Correspondência para:} Fernando Alberto Costa

Cardoso da Silva

Avenida Avicênia,

Condomínio Green Village,

casa 24 - Calhau

São Luís - MA - Brasil

CEP 65071-370

Suporte financeiro: Fundação de Amparo

à Pesquisa e ao

Desenvolvimento Científico

eTecnológico do Maranhão

- FAPEMA.

$O$ referido estudo foi realizado na UFMA.

Os autores declaram a inexistência de conflitos de interesse.

\section{Resumo}

Introdução: A microalbuminúria (MA) e redução da filtração glomerular (FG) podem associar-se às complicações cardíacas da hipertensão arterial (HA), podendo levar a alterações na estrutura, função e geometria do ventrículo esquerdo (VE). Objetivos: Associar alterações da MA e FG com parâmetros do ventrículo esquerdo analisados ao ecodopplercardiograma. Métodos: Realizou-se estudo transversal no período de fevereiro a julho de 2010 com 220 hipertensos tratados em duas unidades de saúde em São Luís. Foram incluídos pacientes $\geq 18$ anos de ambos os sexos. Avaliaram-se dados sociodemográficos, FG e MA em urina de 24 horas, bioquímica, os padrões ecodopplercardiográficos funcionais e geométricos do ventrículo esquerdo. Os pacientes foram agrupados segundo a presença de MA e a taxa de FG. Resultados: O grupo HA com MA apresentou aumento da espessura do VE e índice de massa indexado à superfície corporal (IMSC) e espessura relativa da parede (ERP), menores ondas E' lateral e septal, e maior relação E/e' média $(p<0,05)$. O grupo HA com $\mathrm{FG}<60 \mathrm{~mL} / \mathrm{min} / 1,73 \mathrm{~m}^{2}$ apresentou maiores espessuras, índices de massa e menores relações E/A, menores ondas E' lateral e septal, e maior relação E/e' média $(\mathrm{p}<0,05)$. $\mathrm{O}$ padrão geométrico hipertrofia concêntrica (HVE) apresentou relação com MA $(p=0,002)$. Conclusões: A MA apresentou associação significativa com o aumento das espessuras das paredes do VE e do índice de massa e alterações na função diastólica. Também esteve associada à HVE. A FG apresentou associação significativa com o aumento dos índices de espessuras e massa, e também indicou alterações na função diastólica.

Palavras-chave: Hipertensão. Taxa de Filtração Glomerular. Ecocardiografia.

\section{Abstract}

Introduction: Microalbuminuria (MA) and glomerular filtration rate (GFR) can be associated with cardiac complications hypertension $(\mathrm{AH})$, which may lead to changes in the structure, function and left ventricular geometry. Objectives: To correlate changes of the GFR and MA with the parameters of the left ventricle (LV) assessed by echocardiography. Methods: A cross-sectional study was carried out from February through July 2010 with 220 hypertensive patients treated at two Primary Care Outpatient Clinics in São Luis. Patients $\geq 18$ years of age of both sexes were included in the study. Sociodemographic data, GFR, MA on 24-hour urine, biochemistry and LV geometry and function were assessed. They were divided into two groups according to MA and GFR. Results: The group AH and MA showed increased wall thickness and LV mass indexed for the body surface area (BSA) and relative wall thickness (RWT), lower lateral E' and septal waves, and higher mean E/e' ratio $(\mathrm{p}<0.05)$. The AH group with GFR $<60 \mathrm{~mL} / \mathrm{min} / 1,73 \mathrm{~m}^{2}$ showed increased wall thickness and LV mass indexed for BSA, lower lateral $\mathrm{E}$ 'and septal waves, and higher mean E/e' ratio $(\mathrm{p}<0.05)$. The geometric pattern of LV concentric hypertrophy (LVH) was related to MA ( $p=0.002)$. Conclusions: The MA was significantly associated with increased LV wall thickness and LV mass indexed for BSA, and also changes of diastolic function. Left ventricular concentric hypertrophy was related to MA. The GFR was also significantly associated with increased LV wall thickness and LV mass indexed for BSA suggesting changes of the diastolic function.

Keywords: Hypertension. Glomerular Filtration Rate. Echocardiography. 


\section{INTRODUÇ̃̃O}

A hipertensão arterial sistêmica (HAS) tem prevalência elevada e seu controle ainda não é ideal, constituindo-se em problema de saúde pública no Brasil e no mundo. ${ }^{1}$ No Brasil, há mais de $30 \%$ de hipertensos, ${ }^{2,3}$ dos quais cerca de um terço desconhece sua condição patológica. Dentre aqueles em tratamento, o controle da HAS ainda é considerado baixo. ${ }^{4}$

Os hipertensos usuários do Sistema Único de Saúde (SUS) chegaram em 2006 ao número de 12.410.753.5 Em 2007, houve cerca de 300.000 mortes relacionadas às doenças cardiovasculares (DCV). ${ }^{6}$

A HAS também pode levar a alterações em órgãos-alvo, entre os quais os rins, sendo responsável por cerca de $50 \%$ das inclusões em terapia renal substitutiva (TRS), ${ }^{7}$ o que resultou no Brasil na inclusão de 94.282 novos indivíduos em $2007 .{ }^{1}$ A presença de microalbuminúria (MA) em hipertensos pode levar a um aumento de 2,5 vezes o risco de complicações cardiovasculares, ${ }^{8,9}$ e correlaciona-se significativamente com a gravidade da $\mathrm{HAS}^{10}$. Essa associação foi inicialmente identificada em $1988^{11}$ e em estudos posteriores estabeleceu-se sua importância tanto em hipertensos diabéticos como nos não diabéticos. A MA é considerada um fator de risco independente para complicações cardiovasculares ${ }^{12}$.

A associação entre microalbuminúria, diminuição da filtração glomerular com alterações na geometria e função ventricular esquerda em pacientes hipertensos vem sendo descrita na literatura, ${ }^{13,14}$ podendo contribuir para alterações desfavoráveis nos parâmetros do ventrículo esquerdo, ${ }^{15}$ incluindo pacientes pediátricos ${ }^{16}$ e diabéticos. ${ }^{17}$

Com base nessas observações, o presente estudo teve como objetivo avaliar as associações entre a presença de microalbuminúria, redução da filtração glomerular e alterações morfofuncionais do ventrículo esquerdo em hipertensos atendidos na Atenção Básica de Saúde.

\section{Métodos}

\section{TIPO E LOCAL DO ESTUDO}

Realizou-se um estudo transversal analítico com 220 pacientes hipertensos atendidos na Atenção Básica, cadastrados no Programa HiperDia do Ministério da Saúde, atendidos em dois postos de saúde em São Luís, no Maranhão, no período de fevereiro a julho de 2010.

\section{TAMANHO DA AMOSTRA}

Para se detectar uma diferença de uma unidade $(\mathrm{cm} / \mathrm{s})$ na amplitude da onda E' lateral do anel mitral, considerando-se que a média no grupo de hipertensos sem microalbuminúria foi de $9,0 \mathrm{~cm} / \mathrm{s}$ e no grupo de hipertensos com microalbuminúria de $8,0 \mathrm{~cm} / \mathrm{s}$, com desvio-padrão de 1,7 em ambos, assumindo-se razão de $4: 1$, nível de significância de $95 / \%$ e poder do teste de $80 \%$, seriam necessários 120 pacientes no grupo A e 30 pacientes no grupo B. Foram selecionados hipertensos cadastrados nos postos de saúde no programa HiperDia no período de fevereiro a julho de 2010, localizados pelos respectivos agentes de saúde de cada equipe do Programa Saúde na Família, com idade $\geq$ 18 anos. Os que concordaram em participar assinaram Termo de Consentimento Livre e Esclarecido e foram previamente orientados sobre a coleta de urina de 24 horas, receberam instruções escritas e os recipientes para a coleta. No dia agendado, em jejum, foi coletada amostra sanguínea, amostra simples de urina e recebimento da urina de 24 horas, os quais foram enviados ao laboratório do Hospital Universitário.

Com base na história clínica, exame físico e avaliação ecodopplercardiográfica, adotaram-se os seguintes critérios de não inclusão: portadores de próteses cardíacas, marca-passos, valvopatias, gestantes, coronariopatias, estabelecidas, cardiopatias congênitas, miocardiopatias, arritmias cardíacas, portadores de doença crônica consumptiva (câncer e AIDS) e em hemodiálise.

\section{ColetA de dAdos}

\section{Avaliação clínICA e ANTROPOMÉTRICA}

Os pacientes responderam a um questionário sociodemográfico e de história clínica, e em seguida realizou-se a aferição do peso (em quilogramas) em balança digital portátil (Plenna ${ }^{\circledR}$, São Paulo,Brasil) e da altura (em metros) em estadiômetro (Alturexata ${ }^{\circledR}$, Minas Gerais, Brasil). Diabéticos foram considerados pacientes que assim o declararam ou se encontravam em tratamento.

\section{Avaliação laboratorial}

Para a avaliação laboratorial, foram dosados: glicemia em jejum (método enzimático e valor normal $<99 \mathrm{mg} / \mathrm{dL}$ ) e creatinina sérica (método cinético optimizado U.V. e valor normal $\leq 1,2 \mathrm{mg} / \mathrm{dL}$ ). A filtração glomerular foi avaliada com equação preditiva do Estudo MDRD (Modification of Diet in Renal Disease) ${ }^{18}$ adotando-se os valores $\geq 60 \mathrm{~mL} / \mathrm{min} / 1,73 \mathrm{~m}^{2} \mathrm{e}<60 \mathrm{~mL} / \mathrm{min} / 1,73 \mathrm{~m}^{2}$. 
A microalbuminúria foi determinada em urina de 24 horas pelo método de imunoturbidimetria. Adotaramse como valores de referência o intervalo entre os valores $\geq 30 \mathrm{mg} / 24 \mathrm{~h} \mathrm{e} \leq 300 \mathrm{mg} / 24 \mathrm{~h}$.

\section{AvaliaÇÃo eCODOPPLERCARDIOGRÁFICA}

A avaliação ecocardiográfica foi realizada no prazo de dois dias após a avaliação clínico-laboratorial, pelo mesmo operador, com os pacientes em ritmo sinusal, utilizando-se aparelho da marca General Electric, modelo Vivid 3 (GE Medical Systems, Milwaukee, USA), com segunda harmônica e transdutor eletrônico setorial de dois a quatro MHZ. A interpretação dos dados baseou-se na análise qualitativa e quantitativa das imagens obtidas nos modos unidimensional (modo M), bidimensional, análise do estudo Doppler pulsado, Doppler contínuo e mapeamento de fluxo em cores e Doppler Tecidual. Os valores de referência encontram-se na Tabela 1.

As medidas foram realizadas de acordo com a Sociedade Americana de Ecocardiografia ${ }^{19}$, obtidas diretamente no modo $\mathrm{M}$, com direcionamento pela imagem bidimensional, e incluíram: diâmetro diastólico do VE (DDVE), espessura do septo interventricular (ESIV) e da parede posterior (EPPVE). A fração de ejeção foi medida utilizando-se as medidas lineares do modo $\mathrm{M}$, sendo estimada pela fórmula de Teichholz, e o índice de volume do átrio esquerdo (IVolAE) foi calculado a partir da medida de três diâmetros ortogonais e indexado à superfície corpórea. ${ }^{19}$

A seguinte fórmula foi utilizada para o cálculo da massa do ventrículo esquerdo: $\mathrm{MVE}=0,8\left[1,04(\mathrm{SIV}+\mathrm{DDVE}+\mathrm{PPVE})^{3}-(\mathrm{DDVE})^{3}\right]+0,6 \mathrm{~g} \cdot{ }^{20}$
$\mathrm{O}$ índice de massa foi indexado à superfície corporal (IMSC) e à altura (IM/altura) conforme recomendação da Sociedade Americana de Ecocardiografia. ${ }^{19}$ Em relação à superfície corporal, considerou-se hipertrofia ventricular esquerda (HVE) quando IMSC $>95 \mathrm{~g} / \mathrm{m}^{2}$ para mulheres e $>115 \mathrm{~g} / \mathrm{m}^{2}$ para homens. Com relacão à altura, considerou-se HVE quando IM/altura $>44 \mathrm{~g} / \mathrm{m}^{2,7}$ para mulheres e $>48 \mathrm{~g} / \mathrm{m}^{2,7}$ para homens ${ }^{19}$.

A geometria do ventrículo esquerdo foi classificada em quatro grupos, considerando-se a espessura relativa da parede (ERP $=2 \times P P V E / D D V E)$ e índice de massa relacionada à superfície corporal conforme descrito abaixo. ${ }^{19}$

- Geometria normal: ausência de HVE e espessura relativa $\leq 0,42$.

- Remodelamento concêntrico: ausência de HVE e espessura relativa $>0,42$.

- Hipertrofia excêntrica: presença de HVE e espessura relativa $\leq 0,42$.

- Hipertrofia concêntrica: presença de HVE e espessura relativa $>0,42$.

Para avaliação do estudo Doppler, foram realizadas medidas utilizando-se o Doppler espectral pulsado e tecidual conforme recomendações da Sociedade Americana de Ecocardiografia. ${ }^{21}$ Elas foram obtidas pela média de três ciclos consecutivos com uma velocidade $50 \mathrm{~mm} / \mathrm{s}$. As medidas obtidas na análise do Doppler pulsado em corte apical de quatro câmaras, na ponta dos folhetos mitrais, foram as seguintes: a relação E/A a partir das velocidades de pico das ondas E/A e o tempo de desaceleração da onda E (TDE).

A análise do Doppler Tecidual foi realizada em amostra de volume em anel mitral lateral e septal, em corte apical de quatro câmaras, obtendo-se as

\section{Tabela 1 MEDIDAS ECOCARDIOGRÁFICAS}

\begin{tabular}{lcc} 
Variáveis & Homem & Mulher \\
\hline Índice volume do átrio esquerdo $\left(\mathrm{mL} / \mathrm{m}^{2}\right)$ & 34 & 34 \\
Septo $(\mathrm{mm})$ & 10 & 9 \\
Parede posterior $(\mathrm{mm})$ & 10 & 9 \\
Índice de massa $\left(\mathrm{g} / \mathrm{m}^{2}\right)$ & $\leq 115$ & $\leq 95$ \\
Índice de massa/altura $\left(\mathrm{g} / \mathrm{m}^{2,7}\right)$ & $\leq 48$ & $\leq 44$ \\
Diâmetro diastólico do ventrículo esquerdo $(\mathrm{mm})$ & $<58$ & $<53$ \\
Fração de Ejeção $(\%)$ & $\geq 55$ & $\geq 55$ \\
Onda E' septal (cm/s) & $\geq 8$ & $\geq 8$ \\
Onda E' lateral & $\geq 10$ & $\geq 10$ \\
Relação E/e' média & $<8$ & $<8$
\end{tabular}

Fonte: Lang et al. ${ }^{19}$ e Nagueh et al. ${ }^{21}$ 
medidas de pico das ondas E' em cada anel e a razão onda E fluxo mitral e onda e' do Doppler tecidual (E/e') média, obtida da média aritmética da relação E/e' dos dois anéis. ${ }^{21}$

A função diastólica foi analisada conforme recomendação proposta pela Sociedade Americana de Ecocardiografia, ${ }^{21}$ por meio das análises do Doppler do fluxo mitral, Doppler tecidual e índice de volume do átrio esquerdo. Foi considerada normal quando os pacientes apresentaram onda E' lateral $\geq 10 \mathrm{~cm} / \mathrm{s}$, onda E' septal $\geq 8 \mathrm{~cm} / \mathrm{s}$ e índice de volume do $\mathrm{AE}<34 \mathrm{~mL} / \mathrm{m}^{2}$.

\section{AnÁlise estatística}

Para análise das alterações morfofuncionais do VE com presença de microalbuminúria os pacientes foram agrupados em dois grupos:

- Grupo A: hipertensos sem microalbuminúria.

- Grupo B: hipertensos com microalbuminúria.

Agrupamentos semelhantes foram realizados em relação à taxa de Filtração Glomerular (FG):

- A': hipertensos com $\mathrm{FG} \geq 60 \mathrm{~mL} / \mathrm{min} / 1,73 \mathrm{~m}^{2}$.

- B': hipertensos com $\mathrm{FG}<60 \mathrm{~mL} / \mathrm{min} / 1,73 \mathrm{~m}^{2}$.

Em relação aos padrões geométricos quando relacionados à distribuição urinária da albumina e filtração glomerular, os pacientes foram agrupados em quatro grupos:

- Grupo 1: padrão geométrico normal.

- Grupo 2: remodelamento concêntrico.

- Grupo 3: hipertrofia excêntrica.

- Grupo 4: hipertrofia concêntrica.

As variáveis qualitativas são apresentadas por meio de frequências absolutas e porcentagens, e as quantitativas por média e desvio-padrão quando possuíam distribuição normal e aquelas com distribuição não normal por mediana e amplitude interquartil. Quando a distribuição dos grupos foi normal, eles foram comparados por meio do teste $t$ de Student. Quando as variáveis não apresentaram distribuição normal, foi utilizado o teste de Mann Whitney. Em relação aos padrões geométricos do ventrículo esquerdo e os valores de albumina urinária e filtração glomerular, foi utilizado o teste não paramétrico de Kruskal-Wallis, e quando $\mathrm{p}<0,05$ os grupos foram comparados com o pós-teste de Student-Newman-Keuls. Os dados foram analisados no programa estatístico STATA 11.0. Foi adotado o nível de significância de $5 \%$ em todos os testes realizados.

\section{Aspectos ÉTICOS}

Em observância à Resolução no 196/96 MS/CNS, o presente estudo foi submetido e aprovado pelo Comitê de Ética em Pesquisa do Hospital Universitário sob o protocolo n ${ }^{\circ} 5032 / 2009$ e o parecer $n^{\circ} 185 / 2009$.

\section{Resultados}

Foram avaliados 220 pacientes cadastrados no Programa HiperDia nos dois postos de saúde. Houve predomínio de mulheres $(74,2 \%)$ em relação aos homens $(22,8 \%)$. A mediana de idade foi de $61,23 \pm 11,8$ anos. $\mathrm{O}$ diabetes mellitus encontrava-se presente em 66 pacientes $(29,5 \%)$. A microalbuminúria foi observada em 30 pacientes $(13,4 \%)$ e a filtração glomerular $<60 \mathrm{~mL} / \mathrm{min} / 1,73 \mathrm{~m}^{2}$ ocorreu em 23 pacientes $(10 \%)$.

Os resultados da avaliação ecocardiográfica dos grupos de pacientes hipertensos em relação à microalbuminúria encontram-se na Tabela 2. Observou-se associação significativa $(\mathrm{p}<0,05)$ entre microalbuminúria (grupo B) e maiores valores da ESIV, EPPVE, IMSC e ERP. O IVOLAE, DDVE e IM/altura também foram maiores no grupo $\mathrm{B}$ quando comparados ao grupo A, porém sem significância estatística.

Quando os grupos foram analisados em relação ao Doppler Pulsado, apesar de o grupo B apresentar menores relações E/A, não houve significância estatística. O grupo B, em relação ao Doppler Tecidual, apresentou menores amplitudes das ondas E' septal e lateral e maiores relações E/e' médias do que o grupo $\mathrm{A}(\mathrm{p}<0,05)$.

Em relação à filtração glomerular (Tabela 3), observou-se que a ESIV, EPPVE e o IMSC foram significativamente maiores no grupo B' quando comparados com o grupo A' $(p<0,05)$. Apesar de o grupo B' ter apresentado maiores valores de ERP e IM/altura, não houve diferenças estatisticamente significativas.

Analisando o Doppler Pulsado, evidenciamos que o grupo B' apresentou menores relações E/A $(\mathrm{p}=0,002)$. O grupo B,' ao Doppler Tecidual, apresentou menores amplitudes das ondas E' lateral e septal, e maiores relações E/e' médias $(\mathrm{p}<0,003)$ do que o grupo A'.

Em relação aos padrões geométricos, houve $58,1 \%$ com padrão normal, $8,7 \%$ apresentaram remodelamento concêntrico, 20,9\% hipertrofia excêntrica e $12,3 \%$ padrão de hipertrofia concêntrica.

Quando se analisaram os valores de albumina urinária e o padrão geométrico do ventrículo esquerdo (Tabela 4), observou-se que há uma tendência de a mediana da albumina urinária se deslocar para a direita à medida que a geometria ventricular esquerda 
Tabela 2 AsSOCIAÇÃO ENTRE MEDIDAS ECOCARDIOGRÁFICAS E MICROALBUMINÚRIA EM HIPERTENSOS ATENDIDOS NA Atenção Básica de SAÚde

\begin{tabular}{|c|c|c|c|c|}
\hline Variáveis & $\begin{array}{c}(A) \\
\text { Sem MAB } \\
n=190\end{array}$ & $\begin{array}{c}\text { (B) } \\
\text { Com MAB } \\
\geq 30 \mathrm{mg} / 24 \mathrm{hs} \\
n=30\end{array}$ & $\begin{array}{l}\text { Total } \\
\mathrm{n}=220\end{array}$ & Valor $\mathrm{p}$ \\
\hline Índice EA* & $1,05 \pm 0,27$ & $0,99 \pm 0,24$ & $1,04 \pm 0,27$ & 0,275 \\
\hline Onda $E^{\prime}$ lateral $(\mathrm{cm} / \mathrm{s})^{*}$ & $8,99 \pm 1,65$ & $8,45 \pm 1,93$ & $8,92 \pm 1,69$ & 0,011 \\
\hline Onda $E^{\prime}$ septal $(\mathrm{cm} / \mathrm{s})^{*}$ & $8,01 \pm 1,35$ & $7,33 \pm 1,64$ & $7,92 \pm 1,40$ & 0,016 \\
\hline Média das ondas E/e' lateral e septal $(\mathrm{cm} / \mathrm{s})^{*}$ & $9,40 \pm 1,69$ & $10,50 \pm 2,65$ & $9,59 \pm 1,88$ & 0,002 \\
\hline Média das ondas $\mathrm{E}^{\prime}$ lateral e septal $(\mathrm{cm} / \mathrm{s})^{*}$ & $8,50 \pm 1,42$ & $7,99 \pm 1,73$ & $8,42 \pm 1,47$ & 0,039 \\
\hline Índice de volume do átrio Esquerdo $\left(\mathrm{mL} / \mathrm{m}^{2}\right)^{* *}$ & $18(16-20)$ & $19(17-21)$ & $19(16-20)$ & 0,269 \\
\hline Tempo de desaceleração $(\mathrm{ms})^{*}$ & $229.34 \pm 35,94$ & $234,76 \pm 46,66$ & $230,05 \pm 37,45$ & 0,469 \\
\hline Espessura do septo interventricular $(\mathrm{mm})^{* *}$ & $10(9-10)$ & $10(10-12)$ & $10(9-10)$ & $<0,001$ \\
\hline Espessura da parede posterior $(\mathrm{mm})^{* *}$ & $9(9-10)$ & $10(10-11)$ & $9(9-10)$ & $<0,001$ \\
\hline Fração de ejeção $(\%)^{* *}$ & $63(59-67)$ & $64(60-68)$ & $63(59-67)$ & 0,414 \\
\hline $\begin{array}{l}\text { Diâmetro diastólico do ventrículo Esquerdo } \\
(\mathrm{mm})^{* *}\end{array}$ & $48(45-50)$ & $49(45-51)$ & $48(45-50)$ & 0,367 \\
\hline Índice de massa $\left(\mathrm{g} / \mathrm{m}^{2}\right)^{* *}$ & $90(82-104)$ & $103(95-114)$ & $93(83-106)$ & 0,001 \\
\hline Índice de massa/ altura $\left(\mathrm{g} / \mathrm{m}^{2,7}\right)^{* *}$ & $\begin{array}{c}44,88(38,79- \\
54,21)\end{array}$ & $\begin{array}{c}47,88(40,17- \\
65,31)\end{array}$ & $\begin{array}{c}45,84(38,95- \\
54,55)\end{array}$ & 0,068 \\
\hline Espessura relativa da parede posterior ${ }^{*}$ & $0,40(0,37-0,42)$ & $0,40(0,40-0,44)$ & $0,40(0,38-0,42)$ & 0,020 \\
\hline
\end{tabular}

*Valores representam média \pm desvio-padrão. Valor p para teste $t$ de Student.

**Valores representam mediana Percentil 25-Percentil 75. Valor para o teste de Mann-Whitney.

Índice EA: onda E/onda A; MAB: microalbuminúria.

Tabela 3 AsSOCIAÇÃO ENTRE MEDIDAS ECOCARDIOGRÁFICAS E FILTRAÇÃO GLOMERULAR EM HIPERTENSOS ATENDIDOS NA Atenção Básica de SAÚde

\begin{tabular}{|c|c|c|c|c|}
\hline Variáveis & $\begin{array}{c}\left(\mathrm{A}^{\prime}\right) \\
\mathrm{FG} \geq 60 \mathrm{~mL} / \\
\mathrm{min} / 1,73 \mathrm{~m}^{2} \\
\mathrm{n}=197\end{array}$ & $\begin{array}{c}\left(\mathrm{B}^{\prime}\right) \\
\mathrm{FG}<60 \mathrm{~mL} / \\
\mathrm{min} / 1,73 \mathrm{~m}^{2} \\
\mathrm{n}=23\end{array}$ & $\begin{array}{c}\text { Total } \\
\mathrm{n}=220\end{array}$ & $\begin{array}{c}\text { Valor } \\
\mathrm{p}\end{array}$ \\
\hline Índice E/A* & $1,05 \pm 0,27$ & $0,92 \pm 0,22$ & $1,04 \pm 0,27$ & 0,0024 \\
\hline Onda $E^{\prime}$ lateral $(\mathrm{cm} / \mathrm{s})^{*}$ & $9,01 \pm 1,67$ & $7,73 \pm 1,42$ & $8,88 \pm 1,69$ & $<0,001$ \\
\hline Onda $E^{\prime}$ septal $(\mathrm{cm} / \mathrm{s})^{*}$ & $7,99 \pm 1,36$ & $7,05 \pm 1,53$ & $7,90 \pm 1,40$ & $<0,003$ \\
\hline E/e' média* & $9,47 \pm 1,80$ & $10,15 \pm 2,52$ & $9,54 \pm 1,88$ & $<0,001$ \\
\hline Média das ondas E' lateral e septal $(\mathrm{cm} / \mathrm{s})^{*}$ & $8,54 \pm 1,43$ & $7,34 \pm 1,34$ & $8,42 \pm 1,47$ & $<0,001$ \\
\hline Índice de volume do átrio esquerdo $\left(\mathrm{mL} / \mathrm{m}^{2}\right)^{* *}$ & $19(16-20)$ & $17(13-20)$ & $18(16-20)$ & 0,186 \\
\hline Tempo de desaceleração $(\mathrm{ms})^{*}$ & $230,9 \pm 36,9$ & $234 \pm 46,7$ & $231 \pm 27,9$ & 0,710 \\
\hline Espessura do septo interventricular $(\mathrm{mm})^{* *}$ & $10(9-10)$ & $10(10-12)$ & $10(9-10)$ & 0,013 \\
\hline Espessura da parede posterior $(\mathrm{mm})^{* *}$ & $9(9-10)$ & $10(9-11)$ & $9(9-10)$ & 0,004 \\
\hline Fração de ejeção $(\%)^{* *}$ & $63(59-67)$ & $59(56-65)$ & $63(58-67)$ & 0,131 \\
\hline Diâmetro diastólico do ventrículo esquerdo $(\mathrm{mm})^{*}$ * & $48(45-50)$ & $48(46-51)$ & $48(46-50)$ & 0,539 \\
\hline Índice de massa $\left(\mathrm{g} / \mathrm{m}^{2}\right)^{* *}$ & $91(83-105)$ & $106(94-116)$ & $93(83-108)$ & 0,010 \\
\hline Índice de massa/altura $\left(\mathrm{g} / \mathrm{m}^{2,7}\right)^{* *}$ & 45 (39-54) & $50(39-61)$ & $46(39-54)$ & 0,163 \\
\hline Espessura relativa da parede posterior** & $0,40(0,37-0,42)$ & $0,40(0,39-0,44)$ & $0,40(0,38-0,42)$ & 0,156 \\
\hline
\end{tabular}

* Valores representam média \pm desvio-padrão. Valor p para teste $t$ de Student.

**Valores representam mediana (Percentil 25-Percentil 75). Valor p para o teste de Mann-Whitney.

Índice EA: onda E/onda A; MAB: microalbuminúria; FG: filtração glomerular. 
Tabela 4 AsSOCIAÇÃo ENTRE ALBUMINA URINÁRIA E FILTRAÇÃO GLOMERULAR SEGUNDO OS PADRÕES GEOMÉTRICOS DO Ventrículo esouerdo em hipertensos atendidos na AtençÃo Básica de Saúde

\begin{tabular}{|c|c|c|c|c|c|c|}
\hline Variáveis & $\begin{array}{c}(1) \\
\text { Padrão } \\
\text { normal } \\
(\mathrm{n}=128)\end{array}$ & $\begin{array}{c}(2) \\
\text { Remodelamento } \\
\text { concêntrico } \\
(n=19)\end{array}$ & $\begin{array}{c}(3) \\
\text { Hipertrofia } \\
\text { excêntrica } \\
(\mathrm{n}=46)\end{array}$ & $\begin{array}{c}(4) \\
\text { Hipertrofia } \\
\text { concêntrica } \\
(n=27)\end{array}$ & $\begin{array}{c}\text { Total } \\
(\mathrm{n}=220)\end{array}$ & Valor $p$ \\
\hline $\begin{array}{l}\text { Albumina urinária } \\
(\mathrm{mg} / 24 \mathrm{hs})^{*}\end{array}$ & $6(4-10,5)$ & $6(4-15)^{5}$ & $7(7-15)^{\S}$ & $12(7-31)^{\S}$ & $7(4-13)^{5}$ & 0,002 \\
\hline $\begin{array}{l}\text { Filtração Glomerular } \\
\left(\mathrm{mL} / \mathrm{min} / 1,73 \mathrm{~m}^{2}\right)^{*}\end{array}$ & $\begin{array}{c}84,18(71,51- \\
97,83)\end{array}$ & $\begin{array}{c}79,12(68,73- \\
103,34)\end{array}$ & $\begin{array}{c}89,29(77,95 \\
-102,65)\end{array}$ & $\begin{array}{c}89,53(60,71 \\
-106,35)\end{array}$ & $\begin{array}{c}85,76(71,51- \\
99,34)\end{array}$ & 0,588 \\
\hline
\end{tabular}

*Valores representam mediana (Percentil 25-Percentil 75). Valor p para o teste de Kruskal-Wallis.

${ }^{\S}$ Valor $p$ do pós-teste de Student-Newman-Keuls nos grupos 1 e $4(p=0,002) ; 2$ e $4(p=0,0232) ; 3$ e $4(p=0,0378)$.

varia do normal ao padrão de hipertrofia concêntrica (distribuição assimétrica à direita) com maiores valores no padrão hipertrófico concêntrico $(\mathrm{p}=0,002)$.

Quando comparados em relação à filtração glomerular, não houve diferenças significativas entre os grupos em seus padrões geométricos (Tabela 4).

\section{DIscussão}

O presente estudo teve como objetivo identificar possíveis associações entre microalbuminúria, filtração glomerular e alterações do ventrículo esquerdo, demonstradas pelo ecodopplercardiograma segundo seus padrões de massa, geometria e função em pacientes hipertensos atendidos em unidades de saúde cadastrada no Programa de Atenção Básica, visto que se constituem na "porta de entrada" do paciente quando necessita de atenção em saúde, para posteriormente ser encaminhado a um centro especializado caso seja necessário.

A presença de microalbuminúria em pacientes hipertensos tornou-se nos últimos anos um fator independente de mortalidade e morbidade, motivando novas áreas de pesquisa em doenças cardiovasculares e renais. Tal desordem de filtração de albumina está associada com alterações estruturais e funcionais do ventrículo esquerdo, determinando modificações desfavoráveis nos padrões geométricos, destacando-se as hipertrofias e alterações na função diastólica. Tais alterações podem ocorrer independentemente de alterações na filtração glomerular, especialmente a hipertrofia ventricular esquerda, porém podem vir associadas à sua diminuição. ${ }^{22}$ Neste estudo, observamos que algumas alterações na função diastólica, padrões geométricos e espessura do VE estiveram associados com redução da função renal e presença de microalbuminúria.
A microalbuminúria esteve presente em 30 pacientes $(13,2 \%)$ do total da amostra. Essa prevalência está próxima às encontradas em estudos realizados anteriormente, ${ }^{13,23}$ inclusive em nosso País. ${ }^{24,25}$

Em relação aos achados ecocardiográficos, pudemos observar que os pacientes hipertensos com microalbuminúria apresentaram maiores espessuras das paredes ventriculares e espessura relativa da parede $(\mathrm{p}<0,05)$. Pontremoli, em estudo realizado com 211 pacientes hipertensos não tratados, encontrou associação significativa entre microalbuminúria e aumento das espessuras do septo e parede posterior do ventrículo esquerdo. ${ }^{13}$ Del'Omo, em estudo com 186 pacientes não tratados, encontrou relação entre aumento da espessura relativa da parede e microalbuminúria em seus pacientes. ${ }^{26} \mathrm{O}$ substrato fisiopatológico para essas alterações ainda não se encontra completamente estabelecido, porém processos inflamatórios mediados por microangiopatias, disfunção endotelial no glomérulo e ativação do sistema-renina-angiotensina-aldosterona podem estar implicados. ${ }^{27}$

O grupo de hipertensos com microalbuminúria apresentou maior índice de massa indexada à superfície corporal, dado que também foi encontrado em muitos estudos que associam hipertrofia e microalbuminúria, como Martin et al. em 1996 na Espanha, ${ }^{28}$ incluindo os resultados do estudo LIFE. ${ }^{29}$ Leoncini et al. na Itália também encontraram resultados semelhantes, ${ }^{30}$ e o estudo indiano conduzido por Hitha et al. corroborou esses achados. ${ }^{31}$ Entretanto, quando a microalbuminúria foi associada ao índice de massa indexada à altura, não encontramos em nosso estudo diferença estatisticamente significativa, ao contrário de Kramer et al..$^{32}$ Porém, as diferenças em sua amostra (constituída por $47 \%$ de homens e 
$15 \%$ de diabéticos) comparada a nossa podem ter influenciado nos resultados.

Os dados do estudo Doppler Pulsado evidenciaram que o grupo de hipertensos com microalbuminúria (B) apresentou piores relações E/A que o grupo de hipertensos sem microalbuminúria $(\mathrm{A})$, porém não houve associação estatisticamente significativa. Quando os dados apresentados pelo Doppler Tecidual foram analisados, observou-se que o grupo $\mathrm{B}$ apresentou piores índices de função diastólica caracterizados por menores amplitudes das ondas E' lateral e septal e maiores relações médias E/e' quando comparados com o grupo A com relação estatisticamente significante $(\mathrm{p}<0,05)$, sugerindo que há uma piora na função diastólica. Essas alterações encontradas no Doppler Tecidual guardam semelhanças com os dados encontrados por outros autores, entre os quais Bilge et al., ${ }^{33}$ que, em 2010, num estudo com 105 pacientes, encontraram piora dos índices cardíacos ao Doppler Tecidual associados com níveis de albumina urinária.

Em nosso estudo, também comparamos o grupo de hipertensos com filtração glomerular $<60 \mathrm{~mL} / \mathrm{min} / \mathrm{m}^{2}$ (Grupo B'), que apresentou maiores índices de massa indexada à superfície corporal e maiores espessuras das paredes ventriculares, com o grupo de hipertensos com filtração glomerular $\geq 60 \mathrm{~mL} / \mathrm{min} / \mathrm{m}^{2}$ (Grupo A'). Nardi et al., ${ }^{34}$ utilizando 298 pacientes hipertensos com filtração glomerular normal e 298 pacientes hipertensos com filtração $<89 \mathrm{~mL} / \mathrm{min} / 1,73 \mathrm{~m}^{2}$ (estágio 2) variando até o estágio 5 , encontraram associação entre aumento das espessuras das paredes ventriculares e maiores índices de massa no grupo com diminuição da filtração glomerular, tornando-se inversamente proporcional a ela.

Ao analisarmos os resultados obtidos com o Doppler Pulsado e Doppler Tecidual, observamos critérios que sugeriram disfunção diastólica no grupo de hipertensos com filtração glomerular $<60 \mathrm{~mL} / \mathrm{min} / \mathrm{m}^{2}$. Matsugata et al..$^{35}$ concluíram em um estudo envolvendo 309 pacientes que os índices de disfunção diastólica ao Doppler Tecidual pioravam em pacientes com filtração glomerular diminuída, incluindo um aumento na relação E/e' média, traduzindo aumento nas pressões de enchimento.

Os padrões geométricos do ventrículo esquerdo são adaptações que podem expressar diferente perfil hemodinâmico de cada paciente hipertenso, pois levam em consideração a função ventricular esquerda, a resistência vascular periférica e o débito cardíaco. Esses padrões podem ser avaliados pela ecocardiografia, destacando-se: o remodelamento concêntrico (caracterizado por alta resistência vascular periférica, baixo índice cardíaco e rigidez arterial aumentada); hipertrofia excêntrica (caracterizada por resistência periférica normal e alto índice cardíaco com excesso de volume circulante); e hipertrofia concêntrica (caracterizada por alta pressão sistólica e alta resistência vascular periférica). ${ }^{1}$ O conhecimento das características desses padrões é importante no momento da escolha da medicação anti-hipertensiva. ${ }^{19}$

Em relação à distribuição dos padrões geométricos do ventrículo esquerdo no nosso estudo, houve predomínio do padrão normal em $58 \%$ dos casos seguido pela hipertrofia excêntrica, hipertrofia concêntrica e remodelamento concêntrico em 20,8\%,12,2\% e $9 \%$ respectivamente. Tsioufis et al. ${ }^{15}$ em estudo envolvendo 249 pacientes hipertensos não tratados, encontraram predomínio do padrão normal (34\%), mas um grande número de pacientes com remodelamento concêntrico $(33 \%)$ e menor prevalência de hipertrofia excêntrica com $12 \%$. A hipertrofia concêntrica foi encontrada em $21 \%$.

Quando analisamos os padrões geométricos em relação à excreção urinária de albumina, observamos que a mediana desta foi significativamente maior no grupo com hipertrofia concêntrica do que nos demais grupos, demonstrando que houve associação entre o aumento da excreção urinária de albumina, em especial da microalbuminúria, e esse padrão geométrico conforme descrito em estudos anteriores. ${ }^{13-15} \mathrm{O}$ subestudo MONIKA-KORA ${ }^{36}$ utilizou 1.187 pacientes de uma população geral e concluiu que o aumento de excreção urinária de albumina foi associado com a hipertrofia concêntrica, porém não encontrou associação entre o mesmo padrão geométrico e a filtração glomerular utilizando a equação MDRD. Em nosso estudo, também não encontramos associação estatisticamente significante entre hipertrofia ventricular e filtração glomerular.

A microalbuminúria é um importante fator independente de risco cardiovascular, apresentando associação com a hipertrofia ventricular esquerda. ${ }^{15,16}$ Essa associação também pode ser demonstrada em nosso estudo embora os níveis de microalbuminúria encontrados não tenham sido tão elevados. Essas diferenças podem ocorrer em razão de diferenças nos métodos de análise, população, etnia e coleta. Sabe-se que a microalbuminúria pode ser afetada por obesidade, exercício físico extenuante, consumo excessivo de álcool, cigarro, sobrecarga salina ou proteica, infecção urinária e gravidez. ${ }^{12,25}$

Dentre as limitações do presente estudo, destacam-se em relação aos grupos que não foram abordados 
isoladamente os pacientes portadores de diabetes e soma-se o fato de que os nossos pacientes se encontravam fazendo uso de medicações, enquanto em alguns estudos foram utilizados pacientes sem medicação. ${ }^{13,15}$ Quanto à coleta urinária da microalbuminúria, utilizamos apenas uma amostra enquanto houve estudos que utilizaram de duas a três amostras, embora existam estudos nos quais foi realizada apenas uma coleta com resultados satisfatórios, inclusive utilizando amostra isolada de urina..$^{26,27}$

A relevância desse estudo reside no seu caráter de originalidade em nosso meio e na perspectiva de avaliar as alterações renais em hipertensos na Atenção Básica com melhor custo-efetividade, visto que a filtração glomerular estimada pela equação preditiva utilizou a dosagem da creatinina sérica, exame largamente utilizado inclusive na Atenção Básica, e mostrou-se suficiente para detecção de associação entre a função renal e alterações estruturais e funcionais do ventrículo esquerdo identificadas pelo ecocardiograma.

\section{Conclusão}

O presente estudo permitiu concluir que nos pacientes hipertensos atendidos no Programa de Atenção Básica houve associação entre alterações dos padrões geométricos, em particular da hipertrofia concêntrica do ventrículo esquerdo e do aumento das espessuras das paredes do ventrículo esquerdo, e do seu índice de massa com a microalbuminúria. Também houve associação entre alterações de piora dos índices de função diastólica e a microalbuminúria.

A filtração glomerular diminuída também esteve associada a alterações de aumento das espessuras das paredes do ventrículo esquerdo e índice de massa, tendo apresentado inclusive associação significativa com piora dos índices de função diastólica.

\section{REFERÊNCIAS}

1. Sociedade Brasileira de Cardiologia, Sociedade Brasileira de Hipertensão, Sociedade Brasileira de Nefrologia. VI Diretrizes Brasileiras de Hipertensão. Arq Bras Cardiol 2010;95:1-51.

2. Cesarino CB, Cipullo JP, Martin JPV, et al. Prevalência e fatores sociodemográficos em hipertensos em São José do Rio Preto. Arq Bras Cardiol 2008;91:31-5.

3. Rosário TM, Scala LCNS, França GVA, Pereira MRG, Jardim PCBV. Prevalência, controle e tratamento da hipertensão arterial sistêmica em Nobres, MT. Arq Bras Cardiol 2009;9:672-8.

4. Pereira M, Lunet N, Azevedo A, Barros H. Differences in prevalence, awareness, treatment and control of hypertension between developing and developed countries. J Hypertens 2009;27:963-75.

5. Brasil. Secretaria de Atenção à Saúde. Departamento de Atenção Básica. Hipertensão arterial sistêmica. Ministério da Saúde do Brasil 2006;15:7-14.

6. Malta DC, Moura 1, Sousa FM, Fernandes FM. Doenças crônicas não-transmissíveis: mortalidade e fatores de risco no Brasil, 1990 a 2007 in Saúde Brasil 2008. Brasília: Ministério da Saúde; 2009. p. 337-362.

7. Chobanian AV, Bakris GL, Black HR, et al. The Seventh Report of the Joint national Committee on Prevention, Detection, Evaluation and Treatment of High Blood Pressure: the JNC 7 report. JAMA. 2003;289:2560-72.

8. Tuttle KR. Cardiovascular implications of albuminuria. J Clin Hypertens 2004;6:13-7.

9. Karalliedde J, Viberti G. Microalbuminuria and cardiovascular risk. Am J Hypertens 2004;17:986-93.

10. Cirillo D, Stellato M, Laurenzi W, Panarelli A, Zanchetti N, De Santo G. Pulse pressure and isolated systolic hypertension: association with microalbuminuria. The GUBBIO Study Collaborative Research Group. Kidney Int 2000;58:1211-8.

11. Yudkin JS, Forrest RD, Jackson CA. Microalbuminuria as predictor of vascular disease in non-diabetics subjects. Islington Diabetes Survey. Lancet 1988;2:530-3.

12. Maqueda IG. La microalbuminuria como factor pronóstico em el enfermo cardiovascular. Rev Esp Cardiol 2007;7:31A-43A.

13. Pontremoli R, Ravera M, Bezante GP, et al. Left ventricular geometry and function in patients with essential hypertension and microalbuminuria. J Hypertens 1999;17:993-1000.

14. Pontremoli R, Leoncini G, Ravera M, et al. Microalbuminuria, cardiovascular and renal risk in primary hypertension. J Am Soc Nephrol 2002:13;169-172.

15. Tsioufis C, Stefenadis C, Toutouza M, et al. Microalbuminuria is associated with unfavourable cardiac geometric adaptations in essential hypertensives subjects. J Hum Hypertens 2002;16:249-54.

16. Assadi F. Effect of microalbuminuria lowering on regression of left ventricular hypertrophy in children and adolescents with essential hypertension. Pediatr Cardiol 2007;28:27-33.

17. Picca M, Agozzino F, Pelosi G. Influence of microalbuminuria on left ventricular geometry and function in hypertensive patients with type 2 diabetes mellitus. Ital Heart J 2003;4:48-52.

18. National Kidney Foundation-NKF. K/DOQI clinical practice guidelines for chronic kidney disease: evaluation, classification and stratification. Am J Kidney Dis 2002;39:1-266.

19. Lang RM, Bierig M, Devereux RB, et al. Recommendations for chamber quantification: a report from the American Society of Echocardiography's Guidelines and Standards Committee and the Chamber Quantification Writing Group, developed in conjunction with the European Association of Echocardiography, A Branch of the European Society of Cardiology. J Am Soc Echocardiogr 2005;18:1440-63.

20. Devereux RB, Alonso DR, Lotus EM, et al. Echocardiographic assessment of left ventricular 
ventricular hypertrophy: comparision to necropsy findings. Am J Cardiol 1986;57:450-8.

21. Nagueh SF, Appleton CP, Gillebert TC, et al. Recommendations for the Evaluation of Left Ventricular Diastolic Function by Echocardiography. J Am Soc Echocardiogr 2009;22:107-33.

22. Montelascot G, Collet JP. Preserving cardiac function in the hypertensive patients: why renal parameters hold the key. Eur Heart J 2005;26:2616-22.

23. Toto RD. Microalbuminuria: definition, detection, and clinical significance. J Clin Hypertens 2004;6:2-7.

24. Stamm AMNF, Meinerz G, Silva JC. Hipertensão arterial sistêmica e microalbuminuria. Arq Bras Cardiol 2007;89:415-20.

25. Silva RP, Cisne K, Oliveira JM, Kubrusly M, Sobrinho CRMR, Andrade PJN. Dosagem de microalbuminuria em hipertensos e em pacientes portadores de doença coronariana. Arq Bras de Cardiol 2008;90:108-13.

26. Dell`Omo G, Penno G, Giorgi D, Di Bello V, Mariani M, Pedrinelli R. Association between high-normal albuminuria and risk factors for cardiovascular and renal disease in essential hypertensive men. Am J Kidney Dis 2002;40:1-8.

27. Salles GF, Fiszman R, Cardoso CRL, Muxfeldt ES. Relation of left ventricular hypertrophy with systemic inflammation and endothelial damage in resistant hypertension. Hypertension 2007;50:723-8.

28. Martin M, Lara PA, Lara FJA, et al. Disfunción diastólica, hipertrofia ventricular izquerda y microalbuminuria em la hipertensión arterial esencial ligera-moderada. Rev Esp Cardiol 1997;50:233-37.

29. Watchell k, Palmieri V, Olsen $\mathrm{MH}$, et al. Urine albumin/creatinine ratio and echocardiographic left ventricular structure and function in hypertensive patients with electrocardiographic left ventricular hypertrophy: the LIFE study. Losartan Intervention for Endpoint Reduction. Am Heart J 2002;143: 319-26.

30. Leoncini G, Viazzi F, Conti N, et al. Renal and cardiac abnormalities in primary hypertension. J Hypertens. 2009;27:1064-73.

31. Hitha B, Pappachan JM, Pillai HB, et al. Microalbuminuria in patients with essential hypertension and its relationship to target organ damage: an Indian experience. Saudi J Kidney Dis Transpl 2008;19:411-9.

32. Kramer H, Jacobs DR, Bild D, et al. Urine albumin excretion and subclinical cardiovascular disease. Hypertension 2005;46:38-43.

33. Bilge AK, Atilgan D, Onur I, Pamakcu B, Ozcan M, Adalet K. Relationship between left ventricular hypertrophy, hypertensive retinopathy, microalbuminuria and echocardiographic modalities in newly diagnosed hypertensive patients. Int J Cardiovas Imaging 2010;26:405-12.

34. Nardi E, Palermo A, Mulè G, Cusimano P, Cottone S, Cesarola G. Left ventricular hypertrophy and geometry in hypertensive patients with chronic kidney disease. J Hypertens 2009;27:633-41.

35. Masugata H, Senda S, Goda F, et al. Echocardiographic assessment of the cardio-renal connection: is left ventricular hypertrophy or diastolic function more closely correlated with estimated glomerular filtration rate in patients with cardiovascular risk factors? Clin Exp Hypertens 2010;32:113-20.

36. Lieb W, Stritzke J, Doering A, et al. Association of low-grade albumin excretion with left ventricular hypertrophy in the general population. The MONIKA/ KORA Augsburg Echocardiographic Substudy. Nephrol Dial Transplant 2006;21:2780-7. 